\title{
The diagnostic accuracy of HbA1c in detecting gestational diabetes mellitus among Chinese pregnant individuals
}

\author{
Yi Lai ${ }^{1,2}$, Hanxiao Chen ${ }^{1,3}$, Ze Du ${ }^{3}$, Shu Zhou ${ }^{1,2}$, Wenming Xu ${ }^{1,2,4}$, Tao Li ${ }^{1,2}$ \\ ${ }^{1}$ Department of Gynecology and Obstetrics, West China Second University Hospital, Sichuan University, Chengdu, China; ${ }^{2}$ Key Laboratory of Birth \\ Defects and Related Diseases of Women and Children (Sichuan University), Ministry of Education, Sichuan University, Chengdu, China; ${ }^{3}$ West \\ China School of Medicine, Sichuan University, Chengdu, China; ${ }^{4}$ Joint Laboratory of Reproductive Medicine, Sichuan University-The Chinese \\ University of Hong Kong (SCU-CUHK), West China Second University Hospital, Sichuan University, Chengdu, China \\ Contributions: (I) Conception and design: Y Lai, W Xu, T Li; (II) Administrative support: None; (III) Provision of study materials or patients: None; \\ (IV) Collection and assembly of data: Y Lai, T Li; (V) Data analysis and interpretation: T Li, H Chen; (VI) Manuscript writing: All authors; (VII) \\ Final approval of manuscript: All authors. \\ Correspondence: Tao Li. Department of Gynecology and Obstetrics, West China Second University Hospital, Sichuan University, Chengdu, China. \\ Email: microphage@163.com.
}

\begin{abstract}
Background: This study aims to investigate the diagnostic value of the glycated hemoglobin (HbA1c) test for detecting gestational diabetes mellitus (GDM) in pregnant Chinese patients.

Methods: We enrolled a large cohort of 19,261 pregnant individuals who had both oral glucose tolerance test (OGTT) and HbA1c test between 24 and 28 weeks of gestation in a large Chinese tertiary hospital consecutively from 2013 to 2018. We used Pearson's correlation test to evaluate the correlation between OGTT and HbA1c. The diagnostic accuracy of HbA1c for GDM was examined with the receiver operating characteristic curve, using OGTT as the reference standard.

Results: A total of 3,547 (18.42\%) women were diagnosed with GDM. HbA1c was positively, but only weakly correlated with the fasting, 1-hour glucose, and 2-hour glucose $(r=0.31,0.24$, and 0.25 , respectively, all $\mathrm{P}<0.001)$. The area under the curve of the HbA1c level for detecting GDM was 0.664 (95\% CI: 0.653-0.674, $\mathrm{P}<0.01)$. The optimal cut-off point of $\mathrm{HbA1c}$ for GDM diagnosis was determined at $5.0 \%(31 \mathrm{mmol} / \mathrm{mol})$, which yielded a sensitivity of $60.1 \%$, a specificity of $65.3 \%$, a positive predictive value of $28.1 \%$, and a negative predictive value of $87.9 \%$.
\end{abstract}

Conclusions: HbA1c test is weakly correlated with OGTT during pregnancy, and it offers only limited value in diagnosing GDM among Chinese pregnant individuals.

Keywords: Diabetes; gestational; hemoglobin A; glycosylate; glucose tolerance test

Submitted Jul 02, 2020. Accepted for publication Aug 07, 2020.

doi: $10.21037 /$ atm-20-5464

View this article at: http://dx.doi.org/10.21037/atm-20-5464

\section{Introduction}

Gestational diabetes mellitus (GDM), a common complication of pregnancy, is a severe disease with a prevalence ranging from $<5 \%$ to $20 \%$ (1). Cypryk et al. found that maternal age above 25 years was a risk factor of GDM (2). Many studies reported that there is a strong correlation between women with GDM and adverse pregnancy outcomes, including macrosomia, shoulder dystocia, preeclampsia, increased cesarean rates, as well as maternal and neonatal long-term consequences $(1,3,4)$. GDM patient also has an increased possibility to develop type 2 diabetes mellitus (T2DM) further. Fortunately, treatments for GDM that involve exercising, dietary intervention, plasma glucose monitoring, and insulin therapy are beneficial in reducing adverse pregnancy outcomes $(5,6)$.

The early and exact diagnosis of GDM is of vital importance for pregnant people and their offspring to minimize the risks of adverse events. However, the 
current reference standard of GDM diagnosis relies on a complicated procedure, which presents challenges in implementation, especially in developing countries. Most organizations, including International Association of Diabetes and Pregnancy Study Group (IADPSG) (7), World Health Organization (WHO) (8), and American Diabetes Association (ADA) (9), recommend a one-step method of 2-h 75-g oral glucose tolerance test (OGTT) at 24-28 weeks of gestation. In a standard OGTT, patients must fast for at least $8 \mathrm{~h}$ before the test and take at least three blood samples within $2 \mathrm{~h}$. Due to the complexity of the test, it is challenging to maintain similar standards across different institutions, even more so in some rural areas and developing countries (10).

There is a need for a more simplified and feasible diagnostic procedure for GDM. The WHO used to suggest testing with glycated hemoglobin (HbAlc) with a cutoff point of $6.5 \%$ (48 mmol/mol) for GDM in 2011 (11). However, the $\mathrm{WHO}$ ceased to recommend it as a diagnostic test in 2014 (8). HbA1c test is easy to perform and less invasive, and HbA1c level can reflect the average plasma glucose level of the prior 8-12 weeks. However, various non-conclusive and some controversial studies have been published on the validity of HbA1c in GDM screening and diagnosis. Studies have suggested the relatively low level of HbA1c during pregnancy due to the pregnancy-associated shortened lifespan of red blood cells might limit its value in GDM diagnosis $(12,13)$. Benhalima et al. and Ho et al. demonstrated that HbA1c level of first-trimester and midpregnancy (23-32 weeks) lacks the sensitivity and specificity in the diagnosis of GDM, respectively $(14,15)$.

However, many other studies confirmed the value of HbAlc in GDM diagnosis and suggested different cut-offs for positive cases. A recent meta-analysis confirmed the usefulness of $\mathrm{HbAlc}$ in diagnosing GDM with a pooled area under the curve (AUC) of 0.825 , but they also concluded there was substantial heterogeneity among the studies, with different reported thresholds ranging from $5.4 \%$ to $6.0 \%$ (16). Due to the differences in patients' ethnicity, HbA1c measurement time window, optimal cut-off points, and the reported sensitivity/specificity in different studies, further research of HbA1c in diagnosing GDM in the local patient population is needed to guide clinical practices.

A Thai study analyzed the value of HbA1c in diagnosing GDM, found HbA1c might be a useful tool to reduce the number of OGTT, associated costs and patient inconvenience (17). In that study, the two-step approach was used as the reference standard for GDM. Pregnant women were first screened by $50-\mathrm{g}$ oral glucose challenge tests $(50 \mathrm{~g}$ GCT). Individual who had abnormal $50 \mathrm{~g}$ GCT then underwent a 100-g, 3-h oral glucose tolerance test (100 g OGTT). Pregnant women were diagnosed as GDM if they had two or more abnormal values on the $100 \mathrm{~g}$ OGTT. But in China, one-step method of 75-g, 2-h OGTT is most commonly used to diagnose GDM. For this reason, the diagnostic accuracy of HbA1c in detecting gestational diabetes mellitus among Chinese pregnant individuals remains to be studied.

The present study aims to assess the association between the HbA1c level and glucose levels of OGTT and further determine the diagnostic performance of $\mathrm{HbA1c}$ for GDM, identified by the reference standard of OGTT with ADA/ WHO 2013 criteria. We aim to evaluate the performance of HbA1c in a large sample size to supply evidence and guidance for its potential usage in the diagnosis of GDM. We present the following article in accordance with the STARD reporting checklist (available at http://dx.doi. org/10.21037/atm-20-5464).

\section{Methods}

\section{Study participants}

The study was conducted in accordance with the Declaration of Helsinki (as revised in 2013). This retrospective study was conducted in West China Second University Hospital (WCSUH) by analyzing archived medical records. The Institutional Review Board (IRB) of Sichuan University, West China University Hospital reviewed and approved this study, as it presents a minimum risk to patients with potentially significant benefits for patient care. Given the limited information extracted, the IRB also waived the requirement of informed consent on the individual patient level.

From the archived medical records in WCSUH, we identified all pregnant individuals who had at least one encounter at the outpatient department of obstetrics between January $1^{\text {st }}, 2013$, and October $12^{\text {th }}, 2018$. We selected the first encounter with an OGTT between the $24^{\text {th }}$ and $28^{\text {th }}$ gestation weeks for the cases with multiple encounters. The inclusion process aimed to include a consecutive cohort of pregnant individuals who visited the WCSUH outpatient clinic, as the OGTT was routinely prescribed for pregnant individuals in WCSUH. Patients were excluded if they met any one of the following exclusion criteria: established diagnosis of pre-pregnancy DM or GDM, chronic renal disease, anemia (hemoglobin $<100 \mathrm{~g} / \mathrm{L}$ ), or no $\mathrm{HbAlc}$ measurement at the time of OGTT. 


\section{GDM diagnosis reference standard (OGTT) and index test (HbA1c test)}

The reference standard for GDM diagnosis was from the standardized OGTT according to WHO 2013 criteria which shows that it must meet at least one of the following three criteria: fasting plasma glucose (FPG) $\geq 5.1 \mathrm{mmol} / \mathrm{L}$, 1 -h plasma glucose $(1 \mathrm{~h}-\mathrm{PG}) \geq 10.0 \mathrm{mmol} / \mathrm{L}$, and 2 -h plasma glucose $(2 \mathrm{~h}-\mathrm{PG}) \geq 8.5 \mathrm{mmol} / \mathrm{L}$. We chose WHO 2013 criteria as the reference standard with the fact that it is the most widely recognized and used for GDM diagnosis. After an overnight fast ( $9 \mathrm{~h}$ minimum), patients received a 75-g oral glucose dosage with blood samples collected according to the standardized protocol of OGTT (fasting sample before glucose, 1-h, 2-h sample after glucose) (18). The Hexokinase method measured the venous plasma glucose levels (ADVIA-2400 Chemistry System, Ardmore, Diamond Road, Crumlin, Co Antrim, BT29 4QY, UK) for pregnant individuals. The inter-assay variation for glycemia was $1.57 \%$, and the intra-assay variation was $0.757 \%$.

The index test is the HbA1c measurement in the same sample collected for OGTT after fasting. We used the HPLC method (D-10 Hemoglobin A1c Program, BioRad Laboratories, CA, USA) to measure the HbA1c level in the unit of $\mathrm{mmol} / \mathrm{mol}$. The inter-assay variation for HbA1c was $1.694 \%$, while the intra-assay variation was $1.176 \%$. Given the study aimed to be exploratory rather than focusing on any pre-specified threshold of $\mathrm{HbAlc}$, we collected HbA1cas a continuous measure without any prespecified cut-off point. We aim to analyze the correlation between the continuous measurements of $\mathrm{HbA1c}$ and glucose levels of OGTT and find the optimal cut-off point of HbA1c for GDM diagnosis. Clinical information and reference standard/index test results were unavailable to the performers of the index test/reference standard.

\section{Statistical analysis}

We first compared baseline characteristics of the patients with GDM and subjects without GDM diagnosis. The distributions of continuous variables are shown as the means \pm standard deviations (SDs). Two-tailed $t$-test and the Chi-square test were used to compare the GDM and nonGDM subjects in terms of age, pre-pregnancy body mass index (BMI), systolic blood pressure (SBP), diastolic blood pressure (DBP), family history of diabetes (FHD), previous multiple pregnancies, hemoglobin (HGB), serum ferritin (SF), glucose levels of OGTT and HbA1c level.
As our study aimed to explore the association between OGTT and HbA1c measurements, we evaluated the correlation between the HbA1c and glucose levels of OGTT using Pearson's correlation analysis. The strength of the association, was labeled for absolute values of $r$, $0-0.19$ is regarded as very weak, $0.2-0.39$ as weak, $0.40-0.59$ as moderate, $0.6-0.79$ as strong and $0.8-1$ as very strong correlation. The exploratory nature of the analysis also directed us to utilize the receiver operating characteristic (ROC) curve when examining the performance of the HbA1c measurement in diagnosing GDM at different cutoff points of HbA1c, using OGTT criteria as the reference standard. In addition to the area under the curve (AUC) for the performance, we also calculated the sensitivity, specificity, positive predictive value (PPV), negative predictive value (NPV), and the Youden index (sensitivity + specificity - 1) at different cut-off points of HbA1c. We chose the optimal cut-off point at the value with the maximum Youden index. We produced added performance measures, including the $\mathrm{F} 1$ value and the likelihood ratio for the optimal cut-off point.

All statistical analyses were performed with Jupyterlab version 0.35.4 and Python 3.6.6. A two-sided $\mathrm{P}$ value $<0.05$ was considered statistically significant for all tests.

\section{Results}

\section{Clinical characteristics of participants}

Within the study time, we identified 68,202 pregnant patients who got referred to the outpatient department of obstetrics at WCSUH. After applying the exclusion criteria, we included 63,362 pregnant individuals who underwent an OGTT. However, only 19,261 (30.40\%) received both OGTT and HbA1c test at the same time and thus were included in the association analysis between HbAlc level and glucose levels of OGTT (Figure 1).

Among the 19,261 patients with both the OGTT and HbA1c test, 3,547 pregnant individuals (18.42\%) were diagnosed with GDM following OGTT. Table 1 summarized the clinical and laboratory characteristics of the study participants by disease status.

On average, the GDM patients were older and had higher BMI and more likely to have multiple pregnancies than subjects without GDM diagnosis. More importantly, the mean HbA1c level of pregnant individuals with GDM was significantly higher than that in the non-GDM group $(5.04 \pm 0.38$ vs. $4.82 \pm 0.44, \mathrm{P}<0.05)$. GDM patients also 


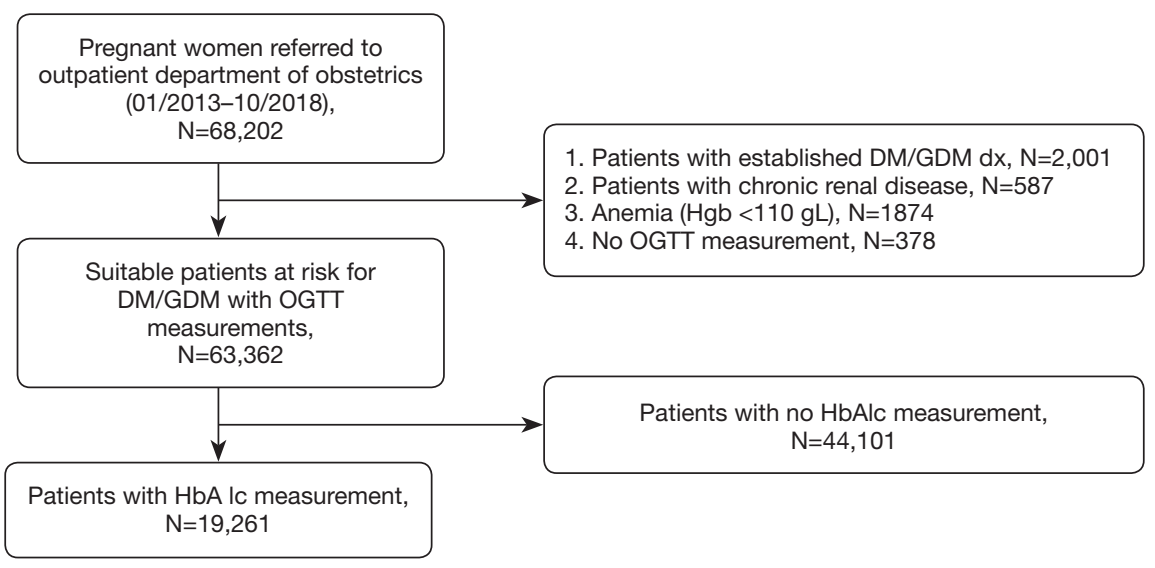

Figure 1 Flow chart of patient selection.

Table 1 Characteristics of pregnant individuals of gestational diabetes mellitus (GDM) and non-GDM groups

\begin{tabular}{|c|c|c|}
\hline Characteristics & GDM group $(n=3,547)$ & Non-GDM group $(n=15,513)$ \\
\hline Age (years) & $32.09 \pm 4.25^{\star}$ & $30.54 \pm 4.08$ \\
\hline Pre-pregnancy body mass index $\left(\mathrm{kg} / \mathrm{m}^{2}\right)$ & $21.31 \pm 2.58$ & $20.83 \pm 2.66$ \\
\hline Systolic blood pressure $(\mathrm{mmHg})$ & $116 \pm 12.3^{*}$ & $110 \pm 11.5$ \\
\hline Diastolic blood pressure (mmHg) & $72 \pm 11.2^{*}$ & $67 \pm 9.6$ \\
\hline Multiple pregnancies (\%) & $4.3 \% *$ & $3.0 \%$ \\
\hline Hemoglobin (g/L) & $122.26 \pm 3.2^{*}$ & $120.17 \pm 4.0$ \\
\hline Serum ferritin $(\mu \mathrm{g} / \mathrm{L})$ & $86.7 \pm 51.2^{*}$ & $67.2 \pm 47.4$ \\
\hline Fasting glycemia (mmol/L) & $4.89 \pm 0.49^{*}$ & $4.43 \pm 0.29$ \\
\hline
\end{tabular}

Data are expressed as mean \pm standard deviation (SD). *, $\mathrm{P}<0.05$ between the GDM group and non-GDM group.

have higher fasting plasma glucose, 1 -h glucose, and $2 \mathrm{~h}$ of glucose (Table 1). GDM patients had slightly higher HGB levels than non-GDM subjects and had significantly higher serum ferritin levels $(86.7 \pm 51.2$ vs. $67.2 \pm 47.4, \mathrm{P}<0.05)$. Far more patients with GDM diagnosis had a prior family history of diabetes $(16.2 \%$ vs. $6.3 \%, \mathrm{P}<0.05)$.

\section{The association between the HbA1c level and glucose levels of OGTT}

To assess the level of correlation between HbA1c, FPG, 1 hPG, and 2 h-PG after OGTT, we calculated the pair wise
Pearson correlation coefficients (Figure 2). Our data proved that there is a strong correlation between $1 \mathrm{~h}-\mathrm{PG}$ and $2 \mathrm{~h}-$ PG $(r=0.71, \mathrm{P}<0.001)$. However, HbA1c was only weakly correlated with the FPG, 1 h-PG, and 2 h-PG after OGTT ( $r=0.31,0.24$ and 0.25 , respectively, all $\mathrm{P}<0.001)$.

\section{The diagnostic performance of $H b A 1 c$ in detecting GDM}

The HbA1c test had low sensitivity and specificity for diagnosing GDM. A ROC curve (Figure 3) was drawn to evaluate HbA1c as a diagnostic tool to detect GDM, and the AUC was 0.664 (95\% CI: 0.653-0.674, $\mathrm{P}<0.01)$. Table 2 


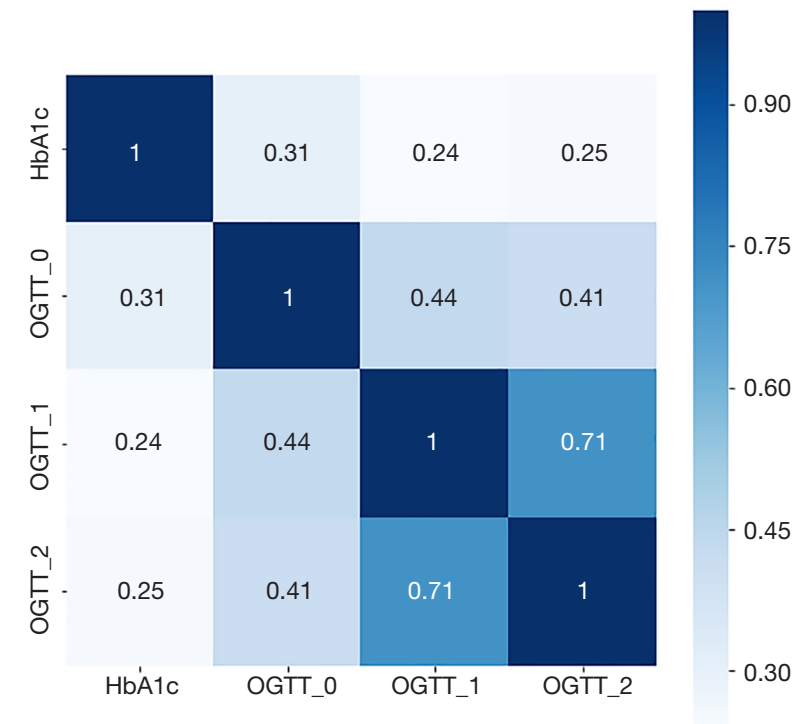

Figure 2 Pearson correlation matrix with numerical coefficients showing a degree of similarity between glycated hemoglobin (HbA1c), oral glucose tolerance test (OGTT) 0 h, OGTT 1 h, and OGTT 2 h levels. The scale shows a color gradient for the correlation coefficient values from 0 to 1 .

shows the specificity, sensitivity, and the Youden index for various cut-off points of HbA1c. The optimal cut-off point, showed by maximizing the sum of sensitivity and specificity, was $5.0 \%(31 \mathrm{mmol} / \mathrm{mol})$ with a sensitivity of $60.1 \%$ and a specificity of $65.3 \%$. According to the optimal cut-off point, $39.4 \%$ of the subjects would be diagnosed with GDM, while the actual disease prevalence was only $18.4 \%$. An alternative cut-off point for HbA1c level of $5.4 \%(35 \mathrm{mmol} / \mathrm{mol})$ showed high specificity of $93.3 \%$ but low sensitivity of $19.7 \%$. On the contrary, the cut-off point of $4.6 \%(27 \mathrm{mmol} / \mathrm{mol})$ presented high sensitivity of $90.6 \%$ but low specificity of $20.7 \%$.

We also noticed that the prevalence of GDM was higher among older women (Table 3). Therefore, we analyzed the performance of $\mathrm{HbAlc}$ in diagnosing GDM in different age groups. Except for women aged 20-24 years, the optimal cutoff point for all other age groups was $5.0 \%(31 \mathrm{mmol} / \mathrm{mol})$, which was the same as the optimal cut-off point. Meanwhile, the optimal cut-off point for each age group also had low sensitivity and specificity for diagnosing GDM (Table 3).

\section{Discussion}

Given the relatively straightforward implementation of HbA1c, even though several studies have questioned the

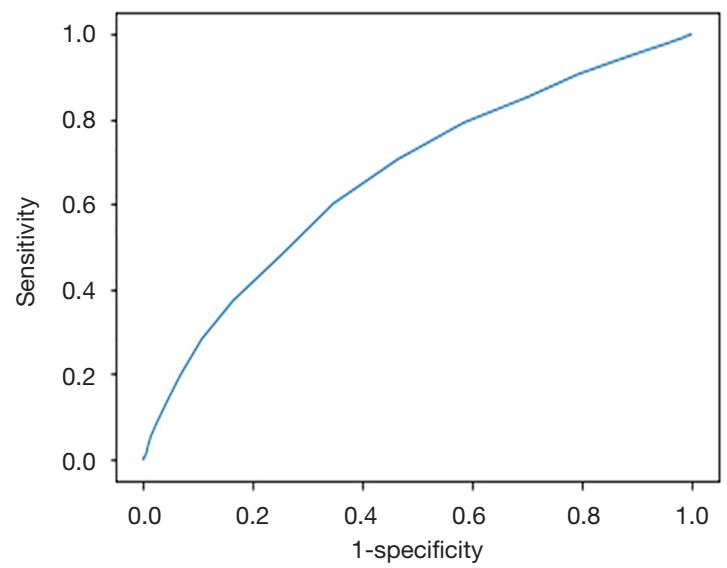

Figure 3 Receiver operating characteristic (ROC) curve analysis of glycated hemoglobin (HbA1c) for diagnosing gestational diabetes mellitus (GDM).

diagnostic value of the HbA1c test for GDM $(14,15,19,20)$, HbA1c was still ordered for pregnant individuals in many institutions, including our outpatient department. The nonconclusive evidence for different optimal cut-off points of HbA1c also contributes to confusion in the application. In this study, we investigated the diagnostic performance of HbA1c and the correlation between HbA1c and traditional OGTT in many pregnant individuals. We expect the evidence generated out of this large sample would clear the above controversies, and provide helpful guidance for utilizing HbA1c in the diagnosis of GDM among Chinese pregnant individuals.

Our study confirmed the differential distribution of HbA1c in Chinese pregnant individuals with or without GDM diagnosis. Our study showed that the average HbA1c level was higher in women with GDM than non-GDM women. To our knowledge, our study is the first research to quantitatively estimate the mean HbA1c value from a large cohort of pregnant individuals with different GDM status. It is essential to note that the average HbA1c level of our study sample is different from other studies done in different racial populations, in which the HbAlc levels tend to be higher. For example, in a Brazilian population, Renz et al. reported that the HbA1c levels of the GDM and nonGDM groups were $5.5 \%$ and $5.1 \%$, respectively (21). We believe that genetic variance may significantly contribute to the race-ethnic difference of the HbA1c level (22).

Although our study had a lower HbA1c level, there is also evidence showing that Asian people may be more susceptible to GDM than Caucasian people $(16,22,23)$. 
Table 2 Diagnostic performance of glycated hemoglobin (HbA1c) test for detecting gestational diabetes mellitus (GDM) at different HbA1c threshold

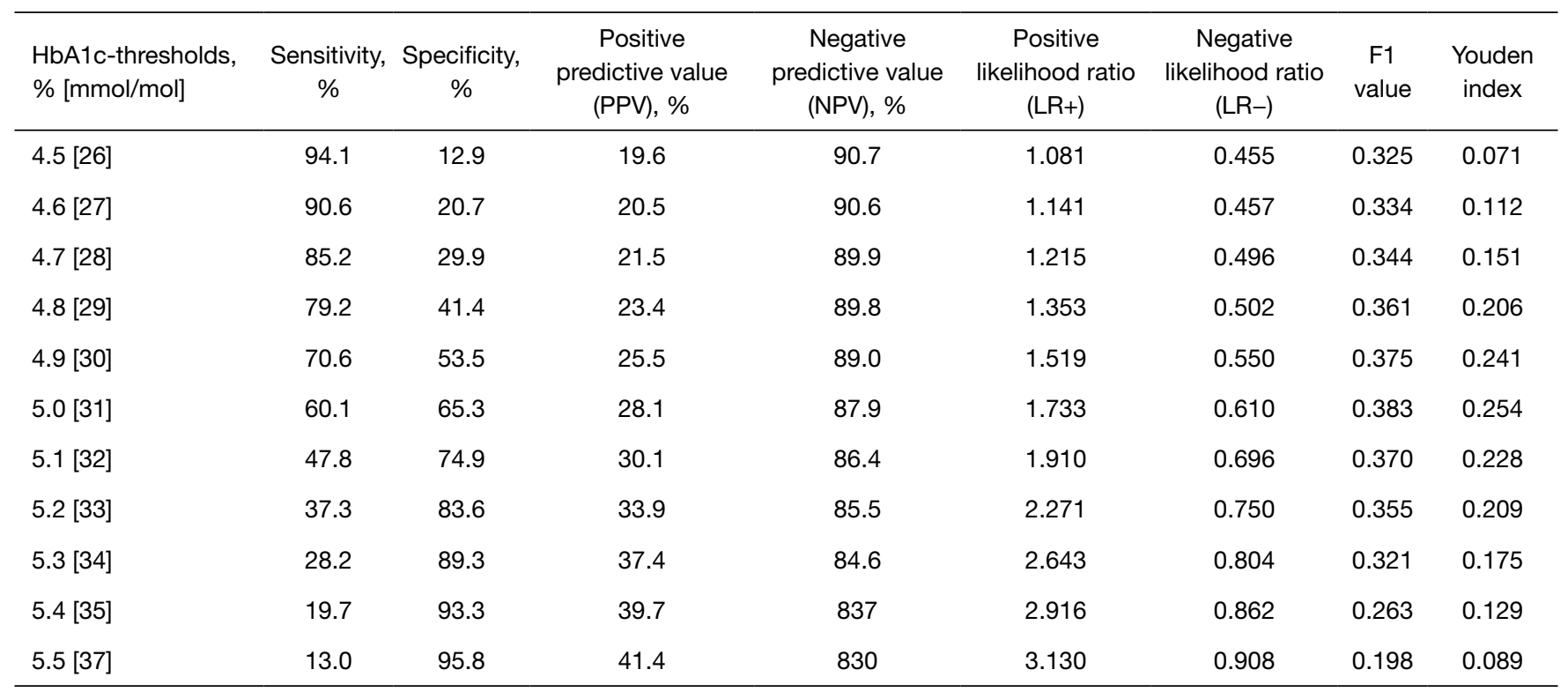

Table 3 Prevalence of gestational diabetes mellitus (GDM)and the optimal cut-off points of glycated hemoglobin (HbA1c) for GDM in different age groups

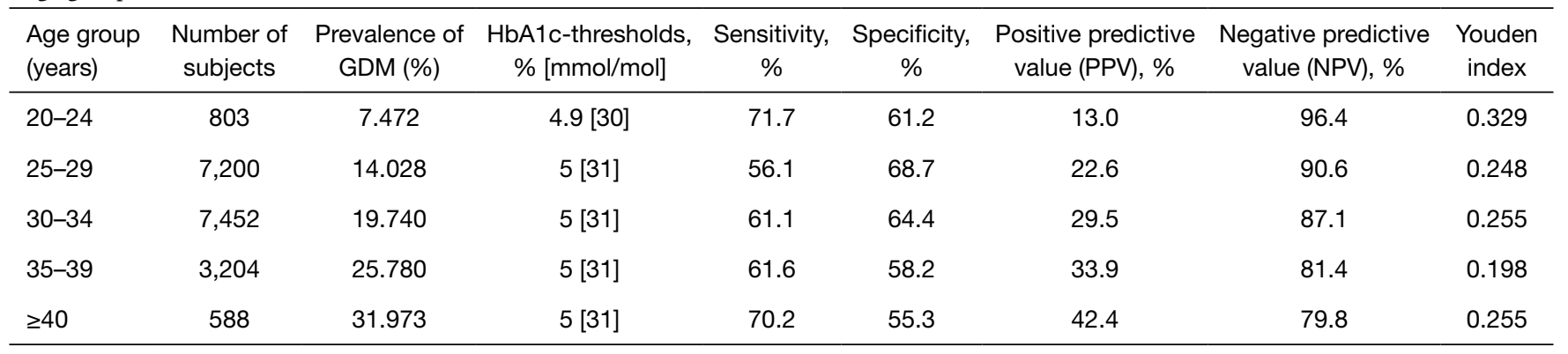

Liu et al. conducted a retrospective study from which they investigated the relationship between ethnicity, age at delivery, and the risk of GDM among 16,258 women in the US (24). According to their results, Asians [OR $=2.81(95 \%$ CI: 2.28-3.48)], Hispanics [OR $=1.27$ (95\% CI: 1.05-1.55)], and Arab Americans [OR =1.46 (95\% CI: 1.20-1.78)] have significantly higher risk and African Americans [OR $=0.64$ (95\% CI: 0.56-0.74)] have lower risk of GDM as compared to whites. Therefore, diagnosing GDM with the optimal cut-off point of HbA1c level obtained from non-Asian studies may not be suitable in the clinical practice in Asian countries.

We found there is a positive but weak correlation between HbA1c value and glucose levels of OGTT, the correlation coefficients between HbA1c and FPG, 1 h-
PG, and 2 h-PG after OGTT is $0.31,0.24$, and 0.25 , respectively. Our results differ from studies that have proved a stronger relationship between the HbA1c test and OGTT. Wang et al. showed that FPG and 2 h-PG were both strongly and positively correlated with $\mathrm{HbAlc}$ $(r=0.83$ and $r=0.80$, respectively). The difference may be attributed to the difference between the population because their subjects were not pregnant (25). We believe our findings of weak correlations between HbA1c and OGTT measurements lay the foundation for further understanding the unique underlying metabolic mechanism of being pregnant.

We further proved the poor diagnostic value of HbA1c for GDM. The AUC of the HbA1c level for detecting GDM was 0.664 (95\% CI: $0.653-0.674, \mathrm{P}<0.01$ ). At the optimal 
cut-off point of $5.0 \%(31 \mathrm{mmol} / \mathrm{mol})$, sensitivity was $60.1 \%$, and specificity was $65.3 \%$, suggesting that HbA1c value is not adequate for detecting GDM. It is noteworthy that our study sample tended to be different from studies done in non-Asian populations, mainly in the distribution of $\mathrm{HbAlc}$. Including the most recent published meta-analysis (16), studies often suggested the optimal cut-off point is $5.4 \%$ or higher, which would yield extremely low sensitivity when applied in our study sample (19.7\%). Even when compared with studies done in similar ethnic populations, our results seemed to favor the HbAlc test, the least, in terms of its diagnostic value for GDM. One Korean study demonstrated that $\mathrm{HbA1c}$ had a sensitivity of $73.6 \%$ and a specificity of $77.2 \%$ at the cut-off point of $5.05 \%(32 \mathrm{mmol} / \mathrm{mol})$ for GDM diagnosis (23).

Meanwhile, in a Taiwan, China study, $5.7 \%(39 \mathrm{mmol} / \mathrm{mol})$ was taken as an optimal cut-off point for detecting GDM with HbA1c. The study also used a sensitivity of $45.2 \%$, and a specificity of $84.1 \%$ (14). However, it must be noted that the Korean study only included 321 patients, while the Taiwan, China study was focused on the high-risk patients who had a positive $50-\mathrm{g}, 1-\mathrm{h}$ glucose challenge test. Our study enrolled a larger sample size without any filtering on the patient baseline risk for GDM. A meta-analysis of pregnant Chinese women also reported the HbA1c performance for detecting GDM. The study revealed it had a sensitivity of $76.2 \%$, a specificity of $91.7 \%$, and an AUC of 0.93 , which was remarkably higher than our estimation (26). However, the studies included in that meta-analysis were outdated, and the sample size of each study was tiny.

Moreover, our findings were like those of another recent sizeable Chinese research, which included 1,959 pregnant individuals, indicating the AUC of HbA1c to detect GDM was 0.66 (95\% CI: $0.63-0.69)$, and the optimal cut-off point of $5.2 \%(33 \mathrm{mmol} / \mathrm{mol})$ could only predict GDM with a sensitivity of $41.6 \%$ and a specificity of $81.5 \%$ (27). Our study also confirmed one of the main findings of the metaanalysis published in 2019 (16). The authors concluded that $\mathrm{HbA1c}$ presented high specificity but low sensitivity regardless of the threshold used. Moreover, we found that older maternal age was associated with a higher prevalence of GDM, consistent with the results of other studies $(24,28)$. However, the diagnostic value of HbA1c in identifying GDM in different age groups remains inadequate.

With the findings of our study, we found only low to moderate accuracy of HbA1c in detecting GDM among Chinese pregnant individuals. The AUC of HbAlc to detect GDM still is inadequate, and the optimal cut-off point for HbAlc is unable to yield both high sensitivity and high specificity simultaneously. We believe the discrepancies could be attributed to multiple factors. First, as mentioned before, the ethnic differences of the population may be crucial to explain the differential distribution of HbA1c and its association with glucose levels of OGTT. Second, although the general protocol of the HbA1c test may have been standardized across many institutions around the world, the exact method of each laboratory may still be different. Lastly, other confounding factors, including comorbid disease, may have a significant impact on the findings. For instance, the prevalence of GDM is reduced in patients with iron deficiency anemia (29).

To our knowledge, this is the study that enrolled the most significant number of participants to evaluate the diagnostic value of $\mathrm{HbA1c}$ for GDM in pregnant Asian women. Evidence generated from our study pointed to the limited diagnostic value of $\mathrm{HbA1c}$ for GDM in this specific patient population. Nevertheless, our research still has some limitations, and the findings should be interpreted with caution. Firstly, patients included in our study were all from West China Second University Hospital, which may not accurately represent other patient populations. Because patients were referred to our large tertiary outpatient center tend to be sicker, the study sample may not represent the patients who reside in less developed regions of China. Secondly, our study only collected few essential characteristics of the patients, which has limited our ability to analyze confounding factors comprehensively. However, we do believe our study in the large patient sample could draw attention to the potential differences between ethnic groups and shed light on the features of Chinese pregnant individuals.

In conclusion, this study confirmed a differential distribution of HbA1c levels between non-GDM and GDM subjects. However, the HbA1c level was only weakly correlated with glucose levels of OGTT. The adoption of HbA1c alone as a diagnostic tool for GDM was not supported by evidence generated from our study. We expect future studies to further examine the diagnostic value of $\mathrm{HbA1c}$ in finely stratified patient subgroups, with proper consideration of all possible confounding factors.

\section{Acknowledgments}

Funding: The Sichuan Science and Technology Program supported this work (grant number: 2019YFS0425), National Key Research and Development Project (grant 
numbers: 2018YFC1002800 and 2018YFC1004603).

\section{Footnote}

Reporting Checklist: The authors have completed the STARD reporting checklist. Available at http://dx.doi.org/10.21037/ atm-20-5464

Data Sharing Statement: Available at http://dx.doi. org/10.21037/atm-20-5464

Conflicts of Interest: All authors have completed the ICMJE uniform disclosure form (available at http://dx.doi. org/10.21037/atm-20-5464). The authors have no conflicts of interest to declare.

Ethical Statement: The authors are accountable for all aspects of the work in ensuring that questions related to the accuracy or integrity of any part of the work are appropriately investigated and resolved. The study was conducted in accordance with the Declaration of Helsinki (as revised in 2013). The Institutional Review Board (IRB) of Sichuan University, West China University Hospital reviewed and approved this study, as it presents a minimum risk to patients with potentially significant benefits for patient care. Given the limited information extracted, the IRB also waived the requirement of informed consent on the individual patient level.

Open Access Statement: This is an Open Access article distributed in accordance with the Creative Commons Attribution-NonCommercial-NoDerivs 4.0 International License (CC BY-NC-ND 4.0), which permits the noncommercial replication and distribution of the article with the strict proviso that no changes or edits are made and the original work is properly cited (including links to both the formal publication through the relevant DOI and the license). See: https://creativecommons.org/licenses/by-nc-nd/4.0/.

\section{References}

1. Kampmann U, Madsen LR, Skajaa GO, et al. Gestational diabetes: A clinical update. World J Diabetes 2015;6:1065-72.

2. Cypryk K, Szymczak W, Czupryniak L, et al. Gestational diabetes mellitus - an analysis of risk factors. Endokrynol Pol 2008;59:393-7.

3. Hyperglycemia and Adverse Pregnancy Outcome (HAPO)
Study Cooperative Research Group. Hyperglycemia and Adverse Pregnancy Outcome (HAPO) study: preeclampsia. Am J Obstet Gynecol 2010;202:255.e1-7.

4. Patti AM, Giglio RV, Pafili K, et al. Pharmacotherapy for gestational diabetes mellitus: still insulin, or what about sulfonylureas? Ann Transl Med 2018;6:S79.

5. Falavigna $M$, Schmidt MI, Trujillo J, et al. Effectiveness of gestational diabetes treatment: a systematic review with quality of evidence assessment. Diabetes Res Clin Pract 2012;98:396-405.

6. Landon MB, Spong CY, Thom E, et al. A multicenter, randomized trial of treatment for mild gestational diabetes. N Engl J Med 2009;361:1339-48.

7. International Association of Diabetes and Pregnancy Study Groups Consensus Panel, Metzger BE, Gabbe SG, et al. International association of diabetes and pregnancy study groups recommendations on the diagnosis and classification of hyperglycemia in pregnancy. Diabetes Care 2010;33:676-82.

8. Diagnostic criteria and classification of hyperglycaemia first detected in pregnancy: a World Health Organization Guideline. Diabetes Res Clin Pract 2014;103:341-63.

9. American Diabetes Association. Standards of medical care in diabetes--2014. Diabetes Care 2014;37 Suppl 1:S14-80.

10. Hod M, Kapur A, Sacks DA, et al. The International Federation of Gynecology and Obstetrics (FIGO) Initiative on gestational diabetes mellitus: A pragmatic guide for diagnosis, management, and care. Int J Gynaecol Obstet 2015;131 Suppl 3:S173-211.

11. Use of Glycated Haemoglobin (HbA1c) in the Diagnosis of Diabetes Mellitus: Abbreviated Report of a WHO Consultation. Geneva: World Health Organization; 2011.

12. Jiao Y, Okumiya T, Saibara T, Park K, Sasaki M. Abnormally decreased HbA1c can be assessed with erythrocyte creatine in patients with a shortened erythrocyte age. Diabetes Care 1998;21:1732-5.

13. Lurie S, Mamet Y. Red blood cell survival and kinetics during pregnancy. Eur J Obstet Gynecol Reprod Biol 2000;93:185-92.

14. Ho YR, Wang P, Lu MC, et al. Associations of midpregnancy $\mathrm{HbA1c}$ with gestational diabetes and risk of adverse pregnancy outcomes in high-risk Taiwanese women. PLoS One 2017;12:e0177563.

15. Benhalima K, Devlieger R, Van Assche A. Screening and management of gestational diabetes. Best Pract Res Clin Obstet Gynaecol 2015;29:339-49.

16. Renz PB, Chume FC, Timm JRT, et al. Diagnostic accuracy of glycated hemoglobin for gestational diabetes 
mellitus: a systematic review and meta-analysis. Clin Chem Lab Med 2019;57:1435-49.

17. Siricharoenthai P, Phupong V. Diagnostic accuracy of $\mathrm{HbA1c}$ in detecting gestational diabetes mellitus. J Matern Fetal Neonatal Med 2019:1-4.

18. Diagnostic criteria and classification of hyperglycaemia first detected in pregnancy: a World Health Organization Guideline. Diabetes Res Clin Pract 2014;103:341-63.

19. Hughes RC, Moore MP, Gullam JE, et al. An early pregnancy $\mathrm{HbA} 1 \mathrm{c} \geq 5.9 \%$ (41 $\mathrm{mmol} / \mathrm{mol})$ is optimal for detecting diabetes and identifies women at increased risk of adverse pregnancy outcomes. Diabetes Care 2014;37:2953-9.

20. Mousavi SN, Kamali K, Mirbazel M, et al. The Best Cut-Off Value for HbA1c as a Screening Tool in Iranian Women With Gestational Diabetes Mellitus. J Family Reprod Health 2017;11:37-42.

21. Renz PB, Cavagnolli G, Weinert LS, Silveiro SP, Camargo JL. HbA1c Test as a Tool in the Diagnosis of Gestational Diabetes Mellitus. PLoS One 2015;10:e0135989.

22. Grimsby JL, Porneala BC, Vassy JL, et al. Race-ethnic differences in the association of genetic loci with HbA1c levels and mortality in U.S. adults: the third National Health and Nutrition Examination Survey (NHANES III). BMC Med Genet 2012;13:30.

23. Kwon SS, Kwon JY, Park YW, et al. HbA1c for diagnosis

Cite this article as: Lai Y, Chen H, Du Z, Zhou S, Xu W, Li T. The diagnostic accuracy of HbAlc in detecting gestational diabetes mellitus among Chinese pregnant individuals. Ann Transl Med 2020;8(16):1014. doi: 10.21037/atm-20-5464 and prognosis of gestational diabetes mellitus. Diabetes Res Clin Pract 2015;110:38-43.

24. Liu B, Lamerato LE, Misra DP. A retrospective analysis of the relationship between race/ethnicity, age at delivery and the risk of gestational diabetes mellitus. J Matern Fetal Neonatal Med 2020;33:2961-9.

25. Wang B, Liu MC, Li XY, et al. Cutoff Point of HbA1c for Diagnosis of Diabetes Mellitus in Chinese Individuals. PLoS One 2016;11:e0166597.

26. Tian QW, Xuan C, Wang HW, et al. Diagnostic accuracy of glycosylated hemoglobin in chinese patients with gestational diabetes mellitus: a meta-analysis based on 2,812 patients and 5,918 controls. Genet Test Mol Biomarkers 2013;17:687-95.

27. Ye M, Liu Y, Cao X, et al. The utility of HbA1c for screening gestational diabetes mellitus and its relationship with adverse pregnancy outcomes. Diabetes Res Clin Pract2016;114:43-9.

28. Bardenheier BH, Imperatore G, Gilboa SM, et al. Trends in Gestational Diabetes Among Hospital Deliveries in 19 U.S. States, 2000-2010. Am J Prev Med 2015;49:12-9.

29. Lao TT, Ho LF. Impact of iron deficiency anemia on prevalence of gestational diabetes mellitus. Diabetes Care 2004;27:650-6.

(English Language Editor: J. Chapnick) 